\title{
Revisión
}

\section{La cicatrización queloide}

(Keloid scarring)

Gustavo Cintrôn-Machón', Juan Poveda-Xatruch²

\section{Resumen}

La cicatriz es el estado final y definitivo de la reparación que el organismo efectúa en una herida accidental, quirúrgica o producida por alguna afección. La cicatrización queloide es un desorden en la estructura normal de la colágena, que conduce a un crecimiento anormal y desproporcionado de las fases normales de la cicatrización, siendo esta un efecto indeseable para todo cirujano. Fue descrita por primera vez en 1.700 a. de J.C. en el papiro de Edwin Smith; sin embargo, no fue hasta en 1802 cuando Alibert usó el término queloide (cheloid=tenaza de cangrejo), por la forma de la lesión. En este artículo se realiza una actualización de esta enfermedad cicatrizal dermoepidérmica, neoplásica.

Descriptores: cicatriz, queloide, colágena

\section{Abstract}

The scarring process is the final and definitive repair of accidental, surgical and other types of wounds. This is a current complete and review of keloid scarring as a dermal - epidermis and neoplasic disease. The physiopathology of keloid scar formation is based on an abnormal collagen structure and alteration of healing phases. Keloid scar is a cosmetic complication undesirable for all surgeons, but it is more important in plastic and reconstructive surgery. On this article we will talk about general aspects, physiopathology and treatment of keloid scars.

Key words: scar, keloid, collagen.

Recibido: 23 de abril de 2007

Aceptado: 9 de octubre de 2007

I Clínica Dr. Carlos Durán Cartín, Caja Costarricense de Seguro Social.

2 Servicio de Cirugía Reconstructiva y Plástica, Hospital Dr. Rafael Ángel Calderón Guardia, Caja Costarricense de Seguro Social Correspondencia: Gustavo Cintrôn Machón. Apartado |442-2 100 Guadalupe

ISSN 0001-6002/2008/50/2/87-93 Acta Médica Costarricense, O2008 Colegio de Médicos y Cirujanos 
Este tipo de cicatrización tiene un crecimiento anormal de sus bordes más allá de la herida original, a diferencia de la cicatrización hipertrófica que se retrae de forma espontánea, ${ }^{2}$ después de ocurrida la lesión (usualmente 4 semanas ${ }^{3}$ ). El queloide deja de crecer varios años después del evento. Se ha observado un tiempo promedio de 30.4 meses. $^{3}$

La cicatrización queloide y la hipertrófica son 2 entidades separadas, que se diferencian clínicamente por el hecho de que la primera crece más allá de la herida original.

Historia: Fue descrita por primera vez en 1.700 a. de J. C. en el papiro de Edwin Smith; ${ }^{4}$ sin embargo, no fue hasta 1802 cuando Alibert usó el término queloide, por la forma de la lesión. ${ }^{3}$ Según un estudio de Marneros y colaboradores, realizado en 341 miembros familiares (14 pedigrees), solo 96 padecían de cicatrización queloide (36 eran de género masculino y 60 de género femenino). La edad y la localización son variables, aunque esta última predomina en el lóbulo de la oreja. Se observó, además, que la herencia en estas familias era del tipo autonómico dominante, con penetrancia clínica incompleta y de expresión variable. Este es el estudio que mejor elucidó las características clínicas genéticas de esta enfermedad. ${ }^{5}$

Se ha determinado que la cicatrización queloide ocurre entre los 10 y los 30 años de edad, y es rara en personas más jóvenes y en los mayores de 50 años. ${ }^{4,6,7}$ También se ha visto que afecta a cualquier etnia, aunque la piel oscura se ve afectada 15 veces más que la tez clara. ${ }^{2,6,8}$ No hay reportes de queloides en albinos., ${ }^{2,11}$ Tiene una frecuencia igual en hombres que en mujeres. ${ }^{9}$ La frecuencia encontrada en la población rural de África es de aproximadamente de un $6,2 \%$ en mujeres y un $5,4 \%$ en hombres. ${ }^{10}$ En población juvenil tiene una alta incidencia, y se presenta en un $12,2 \%$ en el género masculino en y un $14,4 \%$ en el femenino. En la población hispana y negra, Cosman y colaboradores, en una revisión de 3 series largas, notaron la incidencia de la formación de queloide entre un 4,5\% y un $16 \%$, respectivamente. ${ }^{3}$

Las principales causas de la cicatrización queloide son trauma: vacunación y tatuajes, y el arete en el lóbulo de la oreja es el factor predisponente más común. ${ }^{11}$ Dentro de las causas propuestas para la formación anormal cicatrizal están incluidas las reacciones a un cuerpo extraño, infecciones bacterianas, o la posibilidad de degradación o desnaturalización del colágeno. Pero ninguna de estas teorías tienen datos de respaldo. La causa del queloide no es clara. ${ }^{8}$ Existen algunas regiones anatómicas en las que el queloide presenta una incidencia mayor; por ejemplo, la pared anterior del tórax, porque es más dura, diferente a la mama, que tiene una dermis espesa. Los queloides se presentan en áreas donde la concentración de melanocitos es mayor, por lo tanto, son raros en las palmas de las manos y las plantas de los pies, donde la concentración melanítica es menor. ${ }^{12}$
Tratamiento: como en cualquier proceso patológico que no se comprende con claridad, se han aconsejado muchos tratamientos para las cicatrices queloides. ${ }^{16}$ (Cuadro 1)

\section{Excisión}

No se ha logrado un tratamiento clínico óptimo en los queloides. La excisión quirúrgica raramente produce beneficio y solo debe llevarse a cabo después de considerarla con detenimiento. Los queloides tienden a recurrir después de su excisión incompleta, empero, puede haber indicaciones claras para intervenir por medios quirúrgicos en cualquier grupo de pacientes. En algunos los queloides causan tal deformación que puede constituirse en un motivo suficiente para extirpar la lesión. La excisión se utiliza para disminuir su volumen y después se suministran fármacos (ejemplo: corticoesteroides intralesionales) para controlar una cicatriz anormal. En otros pacientes se indica la Excisión con el fin de mejorar la función. Todos los enfermos deben conocer el riesgo alto de nueva formación y la importancia de una vigilancia cuidadosa, ${ }^{10}$ ya que, desafortunadamente, siempre es más grande que la masa primaria.

La excisión por sí sola ha tenido poco éxito en erradicar el queloide. El porcentaje de neoformación ha sido de un $45 \%$ a un $93 \%$. $^{17,18}$

La resección quirúrgica es el método empleado con mayor frecuencia. Sin embargo, si no se acompaña de otras medidas como infiltración de corticoides, presión o radioterapia, fracasará en un altísimo porcentaje de los

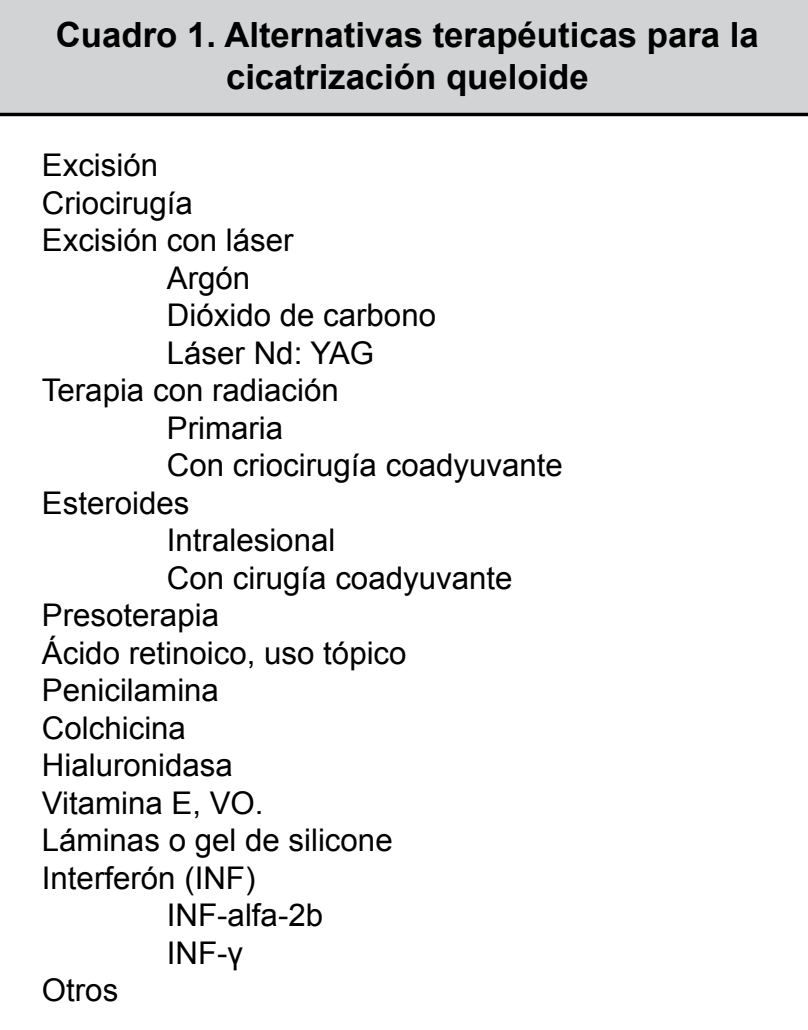


casos. ${ }^{19}$ La cicatrización hipertrófica tiene mejor respuesta a la excisión quirúrgica, mientras que la cicatrización queloide tiende a recurrir. ${ }^{10}$

El hecho de dejar un reborde del queloide no ha mostrado ser un método efectivo. Al resecar el queloide, la sutura no debe quedar tensa. Cuando se remueve un queloide quirúrgicamente, la forma más simple es realizar una incisión elíptica, a la cual siguen 2 semanas o un mes de inyecciones intralesionales de 10 a $40 \mathrm{mg} / \mathrm{ml}$ de acetónido de triamcinolona, y después solo una vez al mes si así se

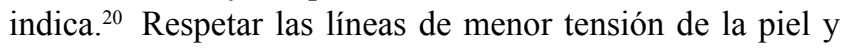
emplear plastías en $\mathrm{Z}$ para relajar la tensión de las cicatrices, disminuye la formación de queloides. Igual efecto pueden tener las suturas intradérmicas. ${ }^{19}$ Al mismo tiempo, el paciente es monitoreado por cualquier efecto indeseable. En la etapa posoperatoria se requiere un compromiso serio del paciente para que la terapia posterior sea un éxito.

La remoción quirúrgica de los queloides en la oreja presenta un único problema terapéutico, ya que es en un pequeño órgano externo del cuerpo, con pocos recursos de agarre tisular para cubrir los defectos quirúrgicos, si una simple incisión elíptica no funcionara. Como consecuencia, se debe crear una aceptable solución cosmética para pasar esto por alto. Sin embargo, se ha reportado que un tercio de los queloides en la oreja pueden ser quitados y reparados para proveer una nueva configuración.

Al efectuar una queloidectomía en la oreja es importante remover el pedazo que está rodeado por el queloide, para evitar las recurrencias. Algunos escojen hacer el simple rasurado del queloide alrededor del lóbulo de la oreja. ${ }^{20}$

\section{Cirugía e irradiación}

Hay diferentes protocolos de radiación que son utilizados. La irradiación posexcisional para prevenir el recrecimiento ha controlado las recurrencias cuando los lugares fueron irradiados dentro de un rango de 600 a 900 Gy. Hubo un reporte de mejoría de hasta el 75\%, al combinar el uso de excisión quirúrgica con irradiación a las 24 horas, en dosis de 1000 a 3000 rad. Las recurrencias más observadas en ese estudio se presentaron en la zona auricular externa, en las personas de piel oscura. ${ }^{20}$

Tepmongkol $^{21}$ estudió pacientes que recibieron radiación (300-500 rad/día por 3 días) con o sin cirugía. El $7.7 \%$ de los pacientes recibieron solamente radiación (11/142), y tuvieron una marcada regresión de la cicatrización queloide. (La regresión no fue precisamente definida.) Los resultados sugieren que no existe beneficio con la irradiación antes de la cirugía. Sin embargo, el uso de una rápida radiación después de la cirugía ha sido más efectivo en la prevención de la recurrencia.

Doornbos y colaboradores ${ }^{22}$ combinaron el uso de cirugía con radiación entre los 900 y los 1500 Gy, siendo las dosis más altas las más efectivas. Observaron un $89 \%$ de recurrencia al año y un $98 \%$ a los 2 años. Se han reportado 3 casos de desarrollo de malignidad luego del tratamiento con radiación, uno en la glándula tiroides y 2 en la glándula mamaria. ${ }^{23,24}$ Estudios en el largo plazo no han documentado una relación entre la malignidad y el grado de radiación.

\section{Radioterapia}

La radioterapia para los queloides ha sido empleada desde 1905 por De Beruman y Gougerot. Gran cantidad de han publicado al respecto. Algunos autores la recomiendan antes y después de la resección quirúrgica del queloide. Otros demostraron que es más efectiva cuando se emplea en el posoperatorio inmediato. ${ }^{3}$ Estudios in vitro han demostrado que la radioterapia produce apoptosis en el fibroblasto queloidiano. ${ }^{25} \mathrm{Su}$ utilización es limitada porque existe el riesgo de inducir malignidad.

La irradiación es más efectiva para el tratamiento de los queloides extensos, pero, para queloides pequeños ha sido debatida. La irradiación es más efectiva que el uso la combinado de excisión y terapia con esteroides. Por la incomodidad asociada al tratamiento intralesional, el paciente se acopla más a la irradiación. ${ }^{20}$ La efectividad de cirugía más irradiación es del 65 al 99\% en el largo plazo.

\section{Otros tipos de irradiación}

a. La irradiación con bomba de electrones no tiene efecto carcinogénico demostrado después del largo plazo; ${ }^{20}$ b. la irradiación ultravioleta A1, tiene un efecto beneficioso en la esclerodermia, según reporte de Asawanonda y colaboradores. ${ }^{26}$ Otros autores aplican esta radiación 3 veces por semana, durante un lapso de 5 a 6 semanas, en 3 pacientes sin tratamientos previos y notan al final de la terapia un leve reblandecimiento del queloide, sin embargo, refieren haber utilizado la mitad de la dosificación de Asawanonda y colaboradores, a lo que obedece que los resultados no fueran satisfactorios. ${ }^{27}$ Esta es una nueva modalidad de tratamiento que necesita más estudios, ya que puede conseguir buenos resultados.

\section{Cirugía con láser}

Las generaciones viejas de ondas continuas de argón neodymium-yttrium-aluminum garnet (Nd:YAG), el láser de dióxido de carbono ( $\mathrm{CO} 2$ láser), han sido usadas para la evaporación de las cicatrices. ${ }^{28-36}$

Desafortunadamente, algunos de estos métodos son rechazados, por la mayor cantidad de recurrencia y de otras secuelas, tales como atrofia de la piel, despigmentación y dolor. Estudios de la década pasada que usaron el "flashlamppumped pulsed dye laser" (PDL) para la cicatrización hipertrófica, demostraron un sorprendente progreso en la textura de la cicatriz. ${ }^{37-43} \mathrm{El}$ mecanismo de funcionamiento del PDL es una termolisis selectiva de las moléculas de la 
hemoglobina, que da como resultado daño microvascular, necrosis de coagulación y, finalmente, hipoxia tisular; y también causa disociación de las bandas de colágeno. ${ }^{44-46}$

El uso de este tipo de láser ha sido exitoso al reducir el color eritematoso de las lesiones y disminuir el riesgo de los efectos adversos. Por lo tanto, el PDL es el tratamiento más popular para la cicatrización hipertrófica y otros tipos de fibrosis, con excepción de que se efectué en las personas negras, debido a la cantidad de melanina, ya que se ha comprobado que el PDL no ejerce buena acción en este tipo étnico.

\section{Crioterapia}

La eficacia de la criocirugía ha sido reportada entre un 50 y un $85 \%,{ }^{47,48}$ sin embargo, se informa también que el nitrógeno líquido destruye los melanocitos, y por lo tanto no es recomendable en personas de piel oscura. ${ }^{20}$ Pierce $^{47}$ fue el primero en utilizar la crioterapia para las cicatrizaciones queloides en el año 1974, pero esta ya había sido utilizada en distintas formas desde 1931.48 Pierce describe su experiencia en 20 pacientes controlados durante un periodo de 2 años. Las cicatrices pequeñas responden bien al tratamiento único en términos de disminución de la cicatrización. En un reporte preliminar, Rousciani y colaboradores ${ }^{49}$ informaron una involución de 48 cicatrices queloides de 64 hombres y mujeres de piel blanca; 2 de 10 pacientes recibieron de $10 \mathrm{a}$ 30 segundos de crioterapia con intervalos de un mes. Zouboulis y colaboradores ${ }^{50}$ examinaron el uso de crioterapia en el tratamiento de cicatrices hipertróficas y queloides en 93 pacientes blancos. Se emplearon diferentes métodos de congelamiento (ambos con óxido nitroso y nitrógeno líquido). El 28\% de los sujetos habían recibido antes otros tratamientos. Los pacientes con los mejores resultados recibieron más de 3 tratamientos. Los efectos secundarios incluyen: dolor, edema, herida infectada, hipoestesia, necrosis, e hipopigmentación. La hipopigmentación puede persistir por muchos años en algunos pacientes.

Shepherd y Dawber ${ }^{51}$ trataron a 17 pacientes con crioterapia. Ellos concluyeron que no se produce cicatriz. Utilizaron nitrógeno líquido como tratamiento y yeso dental para tomar el molde de la cicatriz y controlar la involución. Tres pacientes $(17,5 \%)$ no tuvieron cambios en el volumen; mientras que 7 (41\%) tuvieron una involución mayor del $70 \%$. Tres pacientes negros tuvieron hipopigmentación, la cual se presentó 3 meses después. Los autores dicen que las lesiones pequeñas en el lóbulo de la oreja responden bien a la crioterapia.

Layton y colaboradores ${ }^{46}$ examinaron a 11 pacientes con cicatrización queloide luego de presentar acné, que cursan con múltiples cicatrices queloides en el cuerpo, debido a esa razón. Fueron tratados con crioterapia o triamcinolona $(5 \mathrm{mg} /$ $\mathrm{ml}$ ), y observados por 8 semanas, tras las cuales se concluyó que las lesiones con gran vascularidad responden bien a la crioterapia.

\section{Gel de silicone}

$\mathrm{Su}$ mecanismo de acción no está claro aún. Ha sido utilizado solo, o como terapia adyuvante luego de una escisión, y se ha visto una efectividad luego de 4 a 6 meses de tratamiento. ${ }^{52,53}$ Experimentos in vitro han documentado que el gel de silicone es inerte, no afecta la función del fibroblasto directamente, sino que más bien altera la secreción del factor de crecimiento por parte del queratinocito, lo que provoca una disfunción del fibroblasto y una mala producción de colágeno.

Investigaciones recientes revelan que las láminas de gel de silicone no tienen ningún efecto en la presión, cambio en la temperatura de la cicatrización, $\mathrm{u}$ oxigenación en la tensión de esta. ${ }^{54,55} \mathrm{Su}$ uso produce una disminución en la evaporación de agua en la cicatriz. Más de la mitad es piel normal, ya que el estrato córneo provee el reservorio de líquido. Estas láminas de gel de silicone son impermeables al agua y actúan reduciendo la hemostasis en el estrato corneo, disminuyendo así la hiperemia asociada y la fibrosis. ${ }^{56}$ Se incrementan la hidratación y favorecen el cierre de la lesión.

\section{Interferón}

Los interferones son secretados por las citoquinas producidas por los linfocitos $\mathrm{T}$ (modalidad helper), cuya función es antifibrótica. ${ }^{57,58}$ Este tipo de terapia es experimental cuyos beneficios no han sido bien demostrados. Hay dos tipos de interferón: alfa-2b y gamma. El tipo alfa$2 \mathrm{~b}$ tiene mejores efectos en la modulación del colágeno. ${ }^{59}$ Estudios in vitro sugieren que el interferón modula la actividaddelacolagenasaylasíntesisdeglucosaminoglicanos. Berman y Duncan fueron los primeros en introducir el interferón como tratamiento del queloide en $1989 .{ }^{60}$

Tres estudios con el uso de interferón gamma solo han demostrado una cierta mejoría en la reducción del queloide, $\mathrm{y}$ tres estudios con el uso de interferón alfa-2b, como monoterapia, no demostraron ninguna mejoría. ${ }^{61,62}$

En el estudio de Conejo-Mir y Corbi ${ }^{63}$ se encontró una recurrencia del 33\%, 3 años después de tratamiento con interferón alfa-2b más excisión láser, en 30 cicatrices queloides. Se utiliza de forma intradérmica a través de la lesión, con aplicaciones semanales, pero se concluye que, como monoterapia, no funciona en la remodelación del queloide.

\section{Presoterapia}

Es una técnica que usa vendajes elásticos con tela lycra (hechos sobre medida) o con máscaras de acrílico, aretes especiales, etc. ${ }^{19}$ La presión sostenida ayuda al control del queloide $^{19}$ y su recurrencia. ${ }^{64}$ La presión debe ser superior (sin ser excesiva) a $24 \mathrm{mmHg}$, para superar la capilar, sin llegar a la necrosis. Debe ser continua y sostenida por un lapso superior a los 6 meses. 


\section{Cinco fluoracilo}

Richard Fitzpatrick, el gran estudioso en este tipo de tratamiento, reportó durante sus 9 años de experiencia la administración de más de 5.000 inyecciones, en más de 1.000 pacientes. Cincuenta miligramos por mililitro de 5 fluoracilo (5-FU) fueron inyectados con jeringa alergénica y aguja 27 ó jeringa Luerlock y aguja 30. El 5-FU, a 0,05 ml o menos fue inyectado en intervalos de aproximadamente 1 $\mathrm{cm}$ en la cicatrización. La dosis total administrada se encontró entre 2 y $50 \mathrm{mg}$, y nunca excedió los $100 \mathrm{mg}$ por sesión. Las cicatrices se inflamaron más o se endurecieron más, luego de 3 veces por semana. La frecuencia de inyección era individualizada y acorde con cada paciente. Después la técnica fue refinada y se hizo una combinación de acetónido de triamcinolona $(1 \mathrm{mg} / \mathrm{ml}$ ), con 5-FU (mezcla de $0,1 \mathrm{ml}$ de $10 \mathrm{mg} / \mathrm{ml}$ de acetónido de triamcinolona en $0,9 \mathrm{ml}$ de 50 $\mathrm{mg} / \mathrm{ml}$ de 5-FU), uso del PDL, etc. En el estudio se concluyó que se requieren mejores tratamientos en la cicatrización queloide. ${ }^{65}$

\section{Corticoesteroides}

Tal vez el más popular y eficaz método de tratamiento es la inyección intralesional de triamcinolona, es un fluorocorticoesteroide 9-alfa de acción prolongada. Este hará que la lesión sea más blanda, suave, y con frecuencia más pequeña, y quizá en ello consista la terapéutica necesaria en lesiones menores. Más aún, aliviará el ardor, prurito y dolor relacionados con cicatrices queloides. Si el paciente se queja de estos síntomas somáticos, entonces quizá solo se requieran corticoesteroides, sin importar el tamaño o aspecto de la lesión. Los autores recomiendan $40 \mathrm{mg} / \mathrm{ml}$ de triamcinolona, y no aplicar nunca más de $2 \mathrm{ml}$ cada seis $\mathrm{u}$ ocho semanas en adultos, para evitar cualquier efecto sistémico. Las complicaciones incluyen atrofia local de piel y tejido subcutáneo, que puede ser grave.

Es posible que aparezcan teleangiectasias locales y despigmentación en pacientes de tez oscura. Las mujeres embarazadas nunca deben recibir este tratamiento, por la posibilidad remota de una deformación del nacimiento, relacionada con el uso de corticoesteroides.

Los autores no recomiendan utilizar un "Dermojet" (estas jeringas inyectan en cada disparo $0,1 \mathrm{ml}$ y pueden alcanzar una profundidad de 2 a $5 \mathrm{~mm}$, según la dureza del queloide) para llevar la triamcinolona a través de la epidermis hasta la dermis subyacente. La triamcinolona puede utilizarse aunada a cirugía. Algunos autores la inyectan en los bordes de la herida al momento de cerrar, después de la excisión, en tanto que otros inician el tratamiento en épocas variables después de la operación. Por lo tanto, no hay un manejo estándar, que pueda garantizar la adecuada involución de la cicatriz.
El uso tópico de corticoesteroides no ha sido particularmente efectivo, pero puede ser beneficioso cuando se emplea en combinación con corticoesteroides intralesionales o cirugía.

El mecanismo exacto de acción del corticoesteroide no está todavía claro. El uso de corticoesteroides tópicos o intralesionales se ha asociado a algunos problemas, como: supresión de la comunicación hipotálamo-glándula pituitaria, atrofia de la piel, hipopigmentación, estrías, telangiectasias, foliculitis, exacerbación de la infección preexistente fúngica superficial y acné. También se ha reportado exacerbación de los problemas médicos preexistentes como: Diabetes Mellitus, úlceras intestinales, depresión, rasgos suicidas e hipertensión. Varios tipos de corticoesteroides intralesionales han sido estudiados como monoterapia, y también se han utilizado combinándolos con cirugía.

Darzi y colaboradores ${ }^{66}$ evaluaron diferentes métodos de tratamiento, incluyendo el uso de triamcinolona intralesional. La dosis de administración depende del tamaño de la cicatriz, por lo tanto, queloides de 1 a $2 \mathrm{~cm}^{2}$ se tratan con dosis de 20 a $40 \mathrm{mg}$ de triamcinolona al 0,1\%; los de 2 a $6 \mathrm{~cm}^{2}$, con 60 a $80 \mathrm{mg}$, y los de 6 a $12 \mathrm{~cm}^{2}$, con 80 a 120 $\mathrm{mg}$.

\section{Conclusión:}

La cicatrización queloide es un proceso anormal, donde se encuentra alterada una de sus fases, provocando una variación antiestética, que se convierte en permanente si no es tratada de forma adecuada y oportuna. Su causa no es clara y amerita seguir siendo estudiada.

La importancia de conocer esta anomalía resulta en que el cirujano está propenso a provocarla, no de forma iatrogénica, ya que existen muchos factores que la propician, pero sí es preciso conocer esos agentes para poder prevenir al paciente del riesgo que puede llegar a tener. Según los estudios publicados, no existe un tratamiento ideal en los queloides. Aunque, el uso combinado de radioterapia, crioterapia, inyección intralesional con triamcinolona o la presoterapia, u otros, en vez de la monoterapia, utilizados en un largo periodo (1,5 a 2 años), y valorando constante y cuidadosamente al paciente para evitar los efectos adversos de los tratamientos, llega finalmente a dar un muy buen resultado.

En nuestro país esta entidad es más frecuente de lo que se cree, pues existe una consulta exclusiva para infiltraciones en el Hospital Dr. Rafael Ángel Calderón Guardia. 


\section{Referencias}

1. Breasted JH. The Edwin Smith surgical papyrus: hieroglyphic translation and comentary. Chicago. University of Chicago Press. $1930 ; 403$

2. Murray JC. Scars and keloids. Dermatol Clin. 1993; 11:697.

3. Cosman B, Crickelair GF, Ju MC, Gualin JC, Lattes R. The surgical treatment of keolidal scars. Plast Reconst Surg. 1961; 27:335-358.

4. Urioste SS, Arndt KA, Dover JS. Keloidal scars and hypertrophic scars: review and treatment strategies. Semin Cutan Med Surg. 1999; 18:159-171.

5. Marneros AG. Clinical genetics of familial keloids. Arch Dermatol. 2001; 137:1429-34.

6. Norman B, Bieley HC. Adjunct therapies to surgical management of Keloids. Dermatol Surg. 1996; 2:126.

7. Datubo-Brown DD. Keloids: a review of the literature. Br J Plast Surg. 1990; 43: 70-77.

8. Niessen FB, Spauwen PH, Schalkwijk J, Kon M. On the nature of hypertrophic scars and keloidal scars: a review. Plast Reconstr Surg. 1999; 105: 1435-1458.

9. Mathangi-Ramarkrishnan K, Pothan-Thomas K, Cheyyur R. Study of 1,000 patients with keloidal scars in South India. Plast Reconst Surg. 1974; 53: 276-280.

10. Norris JE. Superficial X-Ray Therapy in Keloid Management: A retrospective study of 24 cases and literatura review. Plast Reconstr Surg. 1995; 95: 1051

11. Oluwasanmi JO. Keloids in the African. Clin Plast Surg. 1974; 1: 179.

12. Rockwell WB, Cohen IK, Ehrlich HP. Keloids and hypertrophic scars: A comprenhensive review. Plast Reconst Surg. 1989; 84:827.

13. Coiffman F. El colágeno inyectable en cirugía plástica. Coiffman F. Cirugía plástica, reconstructiva y estética. Segunda edición. Barcelona: Masson. 1994; 2425.

14. Pollack SV, Gosten JB. The surgical treatment of keloidal scars. J Dermatol Surg Oncol. 1982; 8: 1045-1049.

15. Ackerman AB. Differential diagnosis in dermatolology. Philadelphia \& Febiger. 1982.

16. Schwartz SI, Shires GT, Spencer FC, Fischer JE, Gallowai AC, Daly $\mathrm{JM}$, eds. Principios de Cirugía. $7^{\mathrm{a}}$ ed. México: McGraw Hill Interamericana; 2000 .

17. Lawrence WT. In search of the optimal treatment of keloids: Report of a series and review of the literature. Ann Plast Surg 1991; 27:164.

18. Niessen FB, Sapuwen PH, Shalkwiijk KJ, et al. On nature of hypertrophic scars of 24 cases and literature review. Plast Reconst Surg 1991; 87:50.

19. Coiffman F. Cirugía plástica, reconstructiva y estética. $2^{\mathrm{a}}$ ed. Barcelona: Mason-Salvat; 1994.

20. Errol J. Keloids. Conn's current therapy. Rakel. 54th ed. 799-801.

21. Tepmonkol P. Radiation therapy in the treatment of keloids. J Med Asscoc Thai. 1978; 61:20-5.

22. Doombos JF. The role of kilovoltage irradiation in the treatment of keloids. Int J Radiat Oncol Biol Physiol. 1990; 18:833-9.

23. Botwood N, Lewanski C, Lowdell C. The risk of treating keloids with radiotherapy. Br J Radiol. 1999; 72: 1222-44.

24. Bilbey JH, Muller NL, Miller RR, Nelemus B. Localized fibrous mesothelioma of pleura following external ionizing radiation therapy. Chest. 1988; 94:1291-2.

25. Luo S, Benathan M, Raffoul N, Panizzan RG and Egloff DV. Abnormal balance between proliferation and apoptotic cell death in fibroblasts derived from keloid lesions. Plast Reconst Surg. 2001; 107:87
26. Asawananda P, Khoo LS, Fitzpatrick TB, Taylor CR. UV-A1 for keloid. Arch Dermatol. 1999; 135:348-9.

27. Hannuksela-Svahn A, Grandal OJ, Thorstensen T, Christensen OB UVA 1 for treatment of keloids. Acta Deramtol Venereal. 1999; 79:490.

28. Abergel RP, Dwyer RM, Meeker CA, Uitto J, Lask G, Nelly AP. Laser treatment of keloids: A clinical trial and an in vitro study with $\mathrm{Nd}$ : YAG laser. Lasers Surg Med. 1984; 4:291-295.

29. Apfelberg DB, Maser MR, White DN, Lash H. Failure of carbon dioxide laser excition of keloids. Laser Surg Med. 1989; 9:382-388.

30. Apfelberg DB, Maser MR, Dds HL, White D, Weston J. Preliminary results of argon and carbon dioxide laser treatment of keloid scars. Laser Surg Med. 1984; 4:283-290.

31. Bailin P. Use of the $\mathrm{CO} 2$ laser for non-PWS cutaneous lesions. In: Arndt KA, Noe JM, Rosen S, editors. Cutaneous laser therapy: Principles and Methods. New York: John Wiley; 1983. p. 187-200.

32. Henderson DL, Cromwell TA, Mes LG. Argon and carbon dioxide laser treatment of hypertrophic and keloid scars. Laser Surg Med. 1984;3:271-277.

33. Hulsbergen-Henning JP, Roskam Y. Treatment of keloids and hypertrophic scars with and argon laser. Lasers Surg Med. 1986;6:7275 .

34. Norris JE. The effect of carbon dioxide laser surgery on the recurrence of keloids. Plast Reconstr Surg. 1991;87:44-49.

35. Sherman R, Rosenfeld H. Experience with the Nd:YAG laser in the treatment of keloid scars. Ann Plast Surg. 1988; 21:231-235.

36. Stern JC, Lucente FE. Carbon dioxide laser excision of earlobe keloids. A prospective study and critical análisis of existing data. Arch Otolaryngol Head Neck Surg. 1989; 115:1107-1111.

37. Alster TS. Improvement of erythematous and hypertrophic scars by the 585nm pulsed dye laser. Am Plast Surg. 1994; 32:186-190.

38. Alster TS, Kurban AK, Grove GL, Grove MJ, Tian Tan O. Alteration of argon laser-induced scars by the pulsed dye laser. Lasers Surg Med. 1993; 13:368-373.

39. Alster TS, Lewis AB, Rosenbach A. Laser scar revision: comparison of $\mathrm{CO} 2$ laser vaporization with and without simultaneous pulsed dye laser. Dermatol Surg. 1998; 24:1299-1302.

40. Alster TS, McMeekin TO. Improvement of facial acne scars by the 585nm flashlamp-pumped dye laser. J Am Acad Dermatol. 1996; 35:79-81.

41. Alster TS, Nanni CA. Pulsed dye laser treatment of hypertrophic burn scars. Plast Reconstr Surg. 1998; 102:2190-2195.

42. Alster TS, Williams CM. Treatment of keloid sternotomy scars with $585 \mathrm{~nm}$ flashlamp-pumped pulsed dye laser. Lancet. 1995; 345:11981200.

43. Dierickx C, Goldman MP, Fitzpatrick RE. Laser treatment of erythematous/hypertrophic and pigmented scars in 26 patients. Plast Reconst Surg. 1995; 95:84-90.

44. Aderson RR, Margolis RJ, Watenabe S, Flotte T, Hruza GJ and Dover JS. Selective photo thermolysis of cutaneous pigmentation by Qswitched Nd:YAG laser pulses at 1064, 532 and $355 \mathrm{~nm}$. J Invest Dermatol. 1989; 93:28.

45. Layton AM, Yip J and Cunliffe WJ. A comparison of intralesional triamcinolone and cryosurgery in the treatment of acne Keloids. Br J Dermatol. 1994; 130:498

46. Har-Shai Y, Amar M and Sabo E. Intralesional cryotherapy for enhancing the involution of hypertrophic scars and keloids. Plast Reconst Surg. 2003; 111:1841.

47. Pierce HF. Cryosurgery for hypertrophic scars and keloids. J Natl Med Assoc. 1974; 66:174-5.

48. Lortat-Jacob FJ. Traitement des cheloides par la cryotherapy. Bull Soc Franc Dermatol Et Slip. 1931; 38:979-81.

49. Rusciani L, Rossi G, Bono R. Use of cryotherapy in the treatment of keloids. J Dermatol Surg Oncol. 1993; 19:529-34. 
50. Zouboulis CC, Blume U, Buttner P, Orfanos CE. Outcomes of cryosurgery in keloids and hypertrophic scars. A prospective consecutive trial of case series. Arch Dermatol. 1993; 129:1146-51.

51. Shepherd JP, Dawber RP. The response of keloid scars to cryosurgery. Plast Reconst Surg. 1982; 70:677-81.

52. Sproat JE, Dalcin A, Weitaver N and Roberts RS. Hypertrophic eternal scars: Silicone gel sheet versus kenalog injection treatment. Plast Reconst Surg. 1992; 90:988.

53. Gold MH. A controlled clinical trial of topical silicone gel sheeting in the treatment of hypertrophic scars and keloids. J Am Acad Dermatol. 1994; 30:506.

54. Quinn KG, Evans JH, Courtney JM, Gaylor JDS, Reid WH. Nonpressure treatment of hypertrophic scars. Burns 1985;12:102-8.

55. Quinn KG. Silicone gel in scar treatment. Burns 1987;13:S33-S40.

56. Davey RB. The use of silicone gel and Silastic foam in burn scar management- - how does it work? Presented at the $7^{\text {th }}$ Congress of the International Society for Burn Injuries, Melbourne, Australia, February 1986.

57. Harrop AR, Grahary A, Scout PG, Forsyth N, Uji-Friendhand A and Tredget EE. Regulation of collagen síntesis and mRNA expresión in normal and hypertrophic scar fibroblasts in vitro by interferon-gamma. J Surg Res. 1995; 58:471.

58. Tredget EE, Shankowsky HA, Pannu R, Nedelec B, Iwashina T, Ghahary A, et al. Transforming growth factor-beta in thermally injured patients with hypertrophic scars: Effects of interferon alpha-2b. Plast Reconstr Surg. 1998; 102:1317.
59. Tredget EE, Nedelec B, Scout PG and Garrí A. Hypertrophic scars, keloids and contracturas. The cellular and molecular basis for therapy. Surg Clin North Am. 1997; 77:701.

60. Berman, B., and Duncan, M. R. Short-term keloid treatment in vivo with human interferon alfa- $2 \mathrm{~b}$ results in a selective and persistent normalization of keloidal fibroblast collagen, glycosaminoglycan, and collagenase production in vitro. J. Am. Acad. Dermatol. 21: 694, 1989.

61. Espinassouze F, Heid E and Grosshans E. Treatment of keloid by intralesional injections of interferon alfa-2B (in French). Ann Dermatol Venereol. 1993; 120:629.

62. al Khawajah MM. Failure of interferon-alpha $2 b$ in the treatment of nature keloids. Int J Dermatol. 1996; 35:515.

63. Conejo-Mir JS, Corbi R. Carbon dioxide laser ablation associated with interferon alfa- $2 \mathrm{~b}$ injections reduces the recurrence of keloids. $\mathrm{J}$ Am Acad Dermatol. 1998; 39:1039-40.

64. Pfenninger, Procedures for Primary Care Physicians. 1est edition. Mosby-Year Book. 1994.

65. Fitzpatrick R. Intralesional 5-FU in the treatment of hypertrophic scars and keloids: Clinical Experience. Dermatol Surg. 1999; 25:22432.

66. Darzi MA, Chawdri NA, Kaul SK, Khan M. Evaluation of various methods of treating keloids and hypertrophic scars: a 10 years followup study. Br J Plast Surg. 1992; 45:374-9.

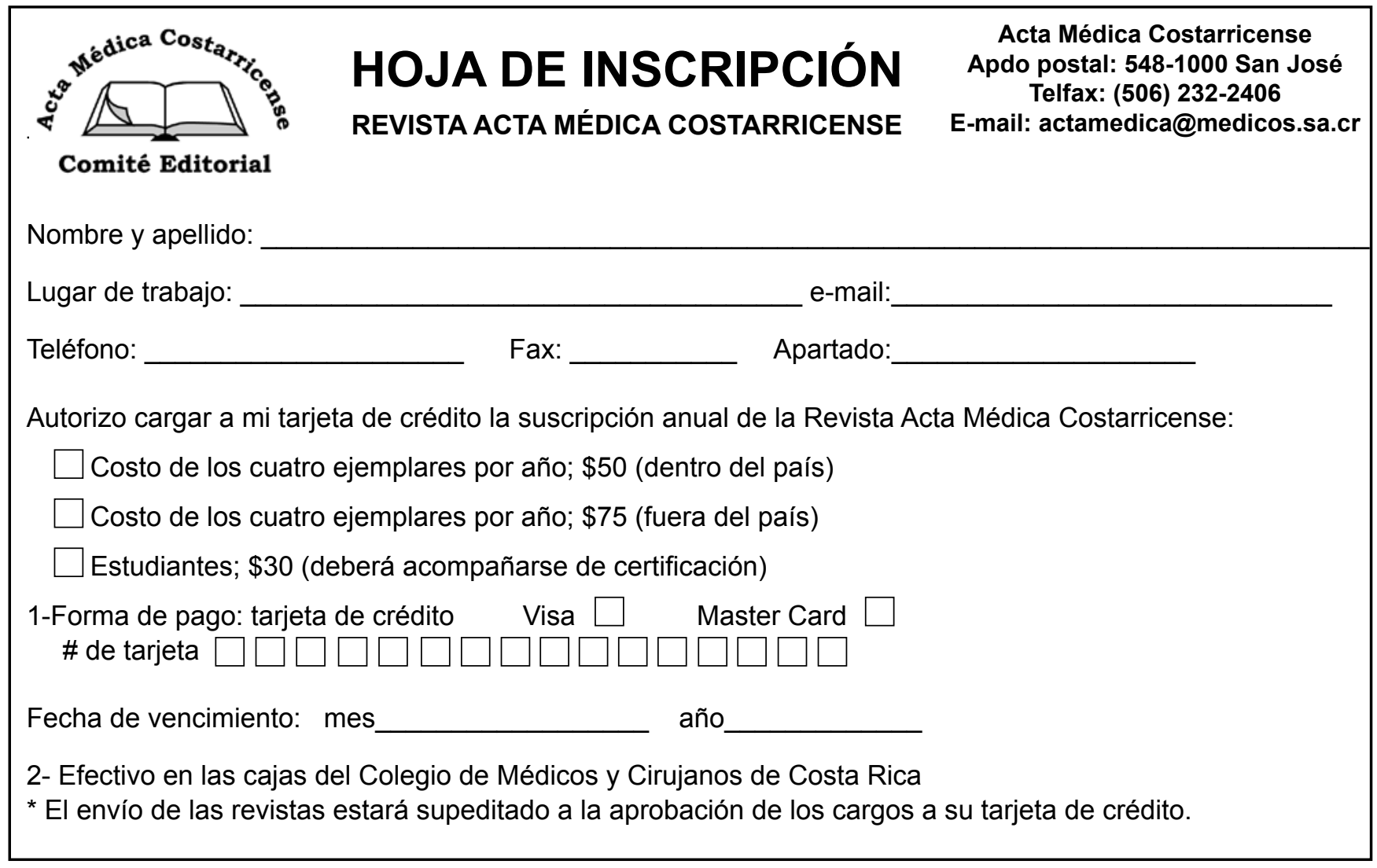

